\title{
Utilizing Superelastic Shape Memory Alloys to Improve the Seismic Response of Bridges
}

\author{
Dr. Shahria Alam \\ The University of British Columbia \\ Vancouver, BC V6T 1Z4, Canada \\ shahria.alam@ubc.ca
}

\begin{abstract}
Shape memory alloy (SMA) is a special kind of material that can undergo large deformations and return to their undeformed shape through stress removal (superelasticity) or heating (shape memory effect). Realizing its enormous potentials in the engineering field, SMAs have been successfully implemented in automotive industries, biomedical devices, and household appliances, etc. SMA's distinct thermomechanical properties and flag-shaped hysteresis also make SMA an ideal contender for the development and design of various structural components for bridge infrastructures in seismic regions. Their performances are expected to supersede conventional structures against seismic hazards as they can potentially recover their deformation even after a major earthquake. This review presents applications of SMAs as reinforcement of structural elements, prestressing applications, and the development of kernel components for seismic devices such as bracings, dampers, and isolators in both new bridge design and construction as well as in deficient bridges for seismic repair/retrofit. Although the high cost of SMAs is still limiting their wide use, research investigating their production and processing will make it more cost-competitive. It is expected that SMAs will emerge as an essential material in the construction industry in near future.
\end{abstract}

